\title{
A simple closure procedure for the study of velocity autocorrelation functions in fluids as a "bridge" between different theoretical approaches
}

V.V. Ignatyuk, a) I.M. Mryglod, and T. Bryk

Institute for Condensed Matter Physics, 1 Svientsitskii Street, 79011, Lviv, Ukraine

Velocity autocorrelation functions (VAF) of the fluids are studied on short- and longtime scales within a unified approach. This approach is based on an effective summation of the infinite continued fraction at a reasonable assumption about convergence of relaxation times of the high order memory functions, which have purely kinetic origin. The VAFs obtained within our method are compared with computer simulation data for the liquid Ne at different densities and the results, which follow from the Markovian approximation for the highest order kinetic kernels. It is shown that in all the thermodynamic points and at the chosen level of the hierarchy, our results agree much better with the MD data than those of the Markovian approximation. The density dependence of the transition time, needed for the fluid to attain the hydrodynamic stage of evolution, is evaluated. The common and distinctive features of our method are discussed in their relations to the generalized collective mode (GCM) theory, the mode coupling theory (MCT), and some other theoretical approaches.

a) Corresponding author: ignat@icmp.lviv.ua 


\section{INTRODUCTION}

Dynamics of many-body systems at various time scales remains as one of the main problems of the non-equilibrium statistical mechanics despite its long history and considerable achievements obtained so far. In the historical retrospective, one should first mention the method of kinetic equations. Though initially this method was designated for the study of the system dynamics, arising from the uncorrelated collisions between two particles in diluted gases $\stackrel{1}{ }$, its subsequent generalizations allow one to describe more realistic systems like dense gases and plasmas².

Nevertheless, it has to be stated that systematic studies of the time behavior of manyparticle systems started with the pioneering work by N.N. Bogoluyubov $\underline{\underline{3}}$, where the concept of weakening correlations allowed one to reformulate a completely unsolvable problem of the $N$-body dynamics into a much more manageable task in terms of the correlation functions of lower orders $s \ll N$.

Within such an approach, a time hierarchy of the physical stages, through which the system passed during its evolution toward equilibrium (starting from the kinetic stage via intermediate steps till the hydrodynamic one and beyond $\underline{4}$ ), appears quite naturally. The corresponding time scales within that hierarchy can serve as a basis for generalized hydrodynamics, and in particular in the generalized collective mode theory $(\mathrm{GCM})^{5}, \underline{6}$, where the set of dynamical variables for description of long- and short-time correlations usually consists of the densities of conserved quantities and their time derivatives up to a certain order. In the framework of the GCM, one actually comes to a simple mathematical problem, expressing collective processes in the system in terms of the dynamic eigenmodes: complex (real) eigenvalues for propagating (relaxing) processes and corresponding eigenvectors which characterize contribution of a particular eigenmode to relevant excitations. The definite advantages of the GCM consist in the facts that: i) the theory describes correctly all the spectrum of collective excitations for different kinds of fluids ${ }^{7-10}$; ii) the theory is a com-

puter adapted one: all the elements of the generalized dynamic matrix could be expressed via the static correlation functions $(\mathrm{SCF})$ and corresponding relaxation times, which can be obtained by molecular dynamic simulations (MD) $\underline{\underline{6}-\underline{\underline{8}}}$; iii) the time correlation functions (TCF) calculated in the framework of the GCM obey the corresponding sum rules (i.e. time expansion series reproduce the results of the exact dynamics up to a ceratin order). 
There is also another method, bearing a close resemblance with the GCM. Using the continued fraction representation $\underline{\underline{11}} \underline{\underline{13}}$ for the Laplace transforms of the VAFs, one can present the corresponding autocorrelation functions (as well as the kinetic kernels of higher orders) as a weighted sum of the exponents. This method operates with an orthogonalized set of the dynamical variables, and the input parameters in such an approach are the SCFs up to the $s$-th order, while hydrodynamic correlation times (the zeroth time moments of the corresponding TCFs), which are inherent to the GCM, are not considered within such a scheme. Though, formally, one can construct the infinite continued fraction, for practical purposes it is necessary to truncate the fraction at the certain level (say, the $s$-th) of the hierarchy. Such an ansatz is related to the Markovian approximation $\underline{\underline{7}}$ for the highest order kinetic kernels (MA), and will be also used in our present paper. Since in both cases the generalized dynamic matrixes are constructed starting from the same conceptual points, there are strong reasons to believe that the results for the TCFs, obtained in the framework of the GCM and within the MA, at the chosen hierarchy level, are close to each other to a high degree of accuracy. This allows one to use the term "mode approximation" for the results obtained within the MAs.

Although the GCM can be extended by taking into account the "ultraslow" processes (defined by the time integrals of corresponding densities $\underline{\underline{14}}-\underline{\underline{16}}$ ), which couple in local approximation with hydrodynamic and extended dynamic variables, usually the problems of account for slow structural relaxation $\underline{\underline{17}}$ are approached by theories with non-local coupling of dynamic variables. In the framework of the mode coupling theory (MCT) $\stackrel{18,19}{\underline{1}}$, a basic set of the dynamic variables consists of higher products ${ }^{20}$ rather than higher derivatives of the densities of conserved quantities. Like in the GCM, a time-spatial dispersion of the kinetic kernels gives rise to some peculiarities of TCF. However, in contrast to the GCM, where the system description at long times is a "bottleneck" of the theory, the MCT approach manifests its efficiency upon the hydrodynamic stage of the system evolution yielding $t^{-3 / 2}$ power law for the $\mathrm{TCFs}^{21}$. In its turn, the $\mathrm{MCT}$ (at least, in its standard formulation $\frac{18}{}$ ) cannot reproduce the sum rules, being unreliable at the description of the fluids dynamics at the short and intermediate times.

Afterwards, a lot of attempts have been made to generalized the MCT, starting from the fundamental works by Götze and co-workers $\underline{22} \underline{\underline{24}}$ up to more recent papers $\underline{25}, 26$. In particular, the main idea of the paper by Szamel $\underline{25}$ was not to factorize immediately the 
memory function expressions leading to the ordinary MCT, but to write the equation of the next hierarchy level, and then factorize the memory function for the new equation. This approach allowed to extend the predictions of the ergodicity breaking transition to higher density cases. In Ref. ${ }^{26}$ the approach based on the truncation of the coupled set of integrodifferential equations for the 2n-point density correlators at high enough level $s=1000$ gave the authors possibility to describe quite perfectly a lot of peculiarities of the "densitydensity" TCFs depending on the generalized bar frequencies and coupling constants, and to put ahead new insights for kinetic theories of the glass transitions.

Very recently, a microscopic interpretation of the long-time tails of VAF was suggested from analysis of its memory kernels 27 . It was shown that the hydrodynamically added mass, defined via the memory kernel, was negative, and the backflow of neighbors tended to drag the particle in the direction of its initial velocity, i.e. contributed negatively to the friction.

The above mentioned theories yield the reliable results for the different time domains: whereas the GCM allows to study the fluid dynamics at the early and intermediate stage of the system evolution, the MCT describes the long-time tails of the corresponding TCF. Moreover, neither GCM nor MCT give possibility to investigate a transition from the intermediate time domain to the hydrodynamic stage. Recently, such an interesting problem has been considered within an approach $\underline{28,29}$ that not only exploits some theoretical schemes, like those above mentioned, but also uses the computer simulation results as the necessary input data for the subsequent complex analysis of the fluids dynamics (therefore, it is sometimes referred to as a "combined" method). In the cited papers, the continued fraction method for the Laplace transforms of the VAFs and reasonable approximations for the memory kernels were used with subsequent analysis of the VAF peculiarities at the complex frequency plane. It was shown that unlike the GCM case, characterized by a limited number of the isolated poles, VAF shows the singularity manifold forming branch cuts. The branch cuts were found to be separated from the real axis by the well-defined "gap". The inverse value of the gap width determines a duration of the transition period from the short-time oneparticle kinetics to the long-time collective motion (hydrodynamics) with a typical power law relaxation $t^{-3 / 2}$, what for VAF has been reported for the first time and explained by Alder and Wainwright ${ }^{30}$.

Summarizing all the above mentioned, we would like to note that in spite of the considerable achievements in exploration of the fluids dynamics, there is still a lack of unified 
self-consistent approaches, being able to describe the system behavior throughout all the stages of its evolution. Thus, an attempt to create and verify such an approach is a motivation of our study. A present paper should be considered as a logical continuation of our very recent article ${ }^{31}$, where the basic concept of the effective summing up of the infinite continued fractions has been formulated, and some relevant theoretical results for the VAFs

behavior have been obtained. However, in Ref. 31 the input parameters of the theory (some kinds of SCFs) had been initially prescribed and did not rely upon the data of experiments or computer simulations.

In the present paper, we take the next step, modelling the dynamics of the realistic fluids. To this end, we perform computer simulations of a number of the autocorrelation functions for the liquid Ne at the temperature $T=295 \mathrm{~K}$ and various densities in the range $\rho=691 \div 2190 \mathrm{~kg} / \mathrm{m}^{3}$. The results obtained within our theoretical scheme are compared with the MD data as well as with those derived by the MA and predicted by the "combined" theories 28,29 .

A structure of the paper is as follows. In Sec. II, we briefly recall the method of continued fractions for the VAF presentation and calculate the kinetic kernels of the highest order at

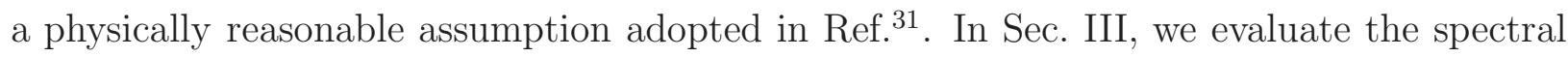
functions both by the effective summation of the continued fraction and in the finite modes approximation. The MD simulation of the VAFs as well as the autocorrelation functions, defined by the higher derivatives of the velocity up to the 4-th order, with a subsequent analysis of the obtained results is a subject of Sec. IV] In Sec. V], we study a time behavior of the VAFs, obtained within different theoretical schemes, and compare the results with the computer simulation data. Finally, we draw the conclusions in Sec. VI.

\section{CONTINUED FRACTION REPRESENTATION FOR VELOCITY AUTOCORRELATION FUNCTION}

To begin with the study of the fluids dynamics, let us consider the normalized VAF

$$
F_{v}(t)=\left\langle\mathbf{v}_{i} \exp \left(-i L_{N} t\right) \mathbf{v}_{i}\right\rangle /\left\langle\mathbf{v}_{i} \mathbf{v}_{i}\right\rangle
$$


where $i L_{N}$ means the Liouville operator. For the system of structureless particles interacting via the central force potential $U\left(\left|\mathbf{r}_{j}-\mathbf{r}_{l}\right|\right)$, it has a quite simple form,

$$
i L_{N}=\sum_{j=1}^{N}\left(\frac{\mathbf{p}_{j}}{m_{j}} \frac{\partial}{\partial \mathbf{r}_{j}}-\sum_{j \neq j=1}^{N} \frac{\partial U\left(\left|\mathbf{r}_{j}-\mathbf{r}_{l}\right|\right)}{\partial \mathbf{r}_{j}} \frac{\partial}{\partial \mathbf{p}_{j}}\right)
$$

with positions $\mathbf{r}_{j}$ and momenta $\mathbf{p}_{j}$ of particles. The Laplace-transformed $\operatorname{VAF} \tilde{F}_{v}(z)=$ $\int_{0}^{\infty} \exp (-z t) F_{v}(t) d t$ obeys the equation

$$
\tilde{F}_{v}(z)=\frac{1}{z+\tilde{\phi}_{1}(z)}
$$

where $\tilde{\phi}_{1}(z)$ are the Laplace-components of the lowest order memory kernel, which contains all the information about the dissipation processes in fluid. What is important for the statement of problem: Eq. (2) is, in fact, an identity like the well-known Ornstein-Zernike equations in the equilibrium theory of the fluids $\frac{32}{2}$. Thus, any specific dynamics of the kinetic kernels like long-time tails (if any noticeable) would manifest itself in the corresponding $\operatorname{VAFs}^{27}$.

An explicit expression for $\tilde{F}_{v}(z)$ can be obtained from the higher order memory kernels $\tilde{\phi}_{j}(z)$, which satisfy a recurrence relation ${ }^{33}$

$$
\tilde{\phi}_{j-1}(z)=\frac{\Gamma_{j-1}}{z+\tilde{\phi}_{j}(z)}
$$

where $\Gamma_{j-1}$ is the relevant SCF. Equations (2)-(3) can be also rewritten in time representation,

$$
\begin{aligned}
& \frac{\partial F_{v}(t)}{\partial t}+\int_{0}^{t} d t^{\prime} \phi_{1}\left(t-t^{\prime}\right) F_{v}\left(t^{\prime}\right)=0, \\
& \cdots \\
& \frac{\partial \phi_{j-1}(t)}{\partial t}+\int_{0}^{t} d t^{\prime} \phi_{j}\left(t-t^{\prime}\right) \phi_{j-1}\left(t^{\prime}\right)=0,
\end{aligned}
$$

where $\phi_{j}(t=0)=\Gamma_{j}$. This is a hierarchy of the generalized Langevin equations, in which the kinetic kernels $\phi_{j}(t)$ are some TCFs of the special kind, defined by the "fluctuating forces" $\underline{34}$.

Using Eqs. (22)-(3), it is possible to present the equation for the Laplace transform $\tilde{F}_{v}(z)$ 
as an infinite continued fraction

$$
\tilde{F}_{v}(z)=\frac{1}{z+\frac{\Gamma_{1}}{z+\frac{\Gamma_{2}}{z+\frac{\Gamma_{3}}{z+\cdots}}}} .
$$

All the SCFs $\Gamma_{j}$ entering Eq. (5) can be calculated according to the general definition ${ }^{33}$. The lowest order SCFs can be presented as follows:

$$
\begin{gathered}
\Gamma_{1}=\frac{1}{m^{2}} \frac{\left\langle\mathbf{F}_{i} \mathbf{F}_{i}\right\rangle}{\left\langle\mathbf{v}_{i} \mathbf{v}_{i}\right\rangle}=\frac{\beta}{3 m}\left\langle\mathbf{F}_{i} \mathbf{F}_{i}\right\rangle, \\
\Gamma_{2}=\frac{\left\langle i L_{N} \mathbf{F}_{i} i L_{N} \mathbf{F}_{i}\right\rangle}{\left\langle\mathbf{F}_{i} \mathbf{F}_{i}\right\rangle}-\Gamma_{1} .
\end{gathered}
$$

In Eqs. (6)-(7), $\mathbf{F}_{i}$ means the force acting on the $i$-th particle, $\mathbf{v}_{i}$ denotes its velocity and the brackets denote ensemble averaging. The "force-force" SCF ([6]) can be evaluated using a pair correlation function of the fluid at a particular thermodynamic point. The SCFs $\Gamma_{j}$ with $j \geq 2$ would require knowledge of even higher order correlation functions, rendering such a problem completely unmanageable. Therefore, the reliable results for the higher order SCFs as well as for the VAFs themselves have to be obtained only by computer simulations (see Sec. IV for the details).

The infinite continued fraction (5) can be rewritten 11 as a weighted sum

$$
\tilde{F}_{v}(z)=\sum_{\alpha=1}^{\infty} \frac{I_{\alpha}}{z-z_{\alpha}},
$$

where $I_{\alpha}$ denote the amplitudes of the particular $\alpha$-th mode of the fluid, whereas $z_{\alpha}$ mean the mode frequencies. Since evaluation of the high order SCFs $\Gamma_{j}$ does not seem a realistic problem from the viewpoint of structural analysis of the fluid or requires time consuming computer simulations, for practical purposes one has to truncate the series (8), retaining only a limited number $M$ of terms $\frac{12}{2}$. In the general case, the issue, at which hierarchy level such a truncation should be performed, has to be solved separately for each particular system and for each particular thermodynamic point $\stackrel{12,13}{ }$.

A simple truncation of the series (8), even at large number of terms, does not allow to describe the system dynamics exactly, especially at long times $\underline{18}, \underline{19}$. On the other hand, from a strictly mathematical viewpoint, there is no other reason for the power law behavior of the VAFs at large $t$, but a contribution of the infinite number of the terms in (8) $)$. Therefore, 
it would be challenging to propose a self-consistent theory that could reliably describe the fluids dynamics within the whole time domain with: i) taking advantages of the GCM or MA approaches, and ii) its application for the long times at the expense of the properly designed kinetic kernels.

To this end, let us make an important remark. The Markovian approximation of the lowest order kinetic kernel is known $n^{\frac{35}{5}}$ to determine the relaxation time $\tau_{1}$ of the corresponding hydrodynamic excitation by a simple relation $\tilde{\phi}_{1}(0)=\tau_{1}^{-1}$. Further natural step would be an introduction (in a similar manner) of the higher order relaxation times, $\tau_{j}$, with $j \geq 2$. It is known from the theory of continued fractions $\frac{36}{3}$ as well as from the analysis of fluid dynamics 37 that $\tau_{j}$ oscillate around a certain value $\tau^{*}$ depending on the level of description, and approach this asymptotics from below (odd order relaxation times) or above (even order relaxation times). Moreover, the difference between $\tau_{2 j}$ and $\tau_{2 j+1}$ decreases rapidly with increasing of the hierarchy level $j$. A key point of our approach is a physically grounded assumption that the relaxation times of the high order kinetic kernels become comparable with each other and, starting from the order $s$, tend to a certain fixed value, which has a purely kinetic origin. In this context, our assumption is in a close agreement with the results 10,37 for characteristic times of decay of the autocorrelation functions of simple and polar fluids, obtained by an approximated summation of the continued fractions.

The above formulated statement can be formalized in the following way. Let us suppose that the memory kernels $\tilde{\phi}_{j}(z)$ tend to a certain (limiting) function with the increase of $j$. If we suppose this convergence to occur for two kinetic kernels of the neighboring orders, starting from large enough $j=s$,

$$
\tilde{\phi}_{s}(z) \approx \tilde{\phi}_{s+1}(z),
$$

then the recurrence relation (3) converts into a bilinear form, which gives us immediately the explicit expression for the highest order memory kernel

$$
\tilde{\phi}_{s}(z)=-\frac{z}{2}+\sqrt{\frac{z^{2}}{4}+\Gamma_{s}} .
$$

The relation (9) is, in fact, the $s$-th order approximation for the memory kernels at the chosen ansatz. In contrast to the Markovian approximation,

$$
\tilde{\phi}_{s}(z) \approx \tilde{\phi}_{s}(0),
$$


when the real part of the kinetic kernel $\tilde{\phi}_{s}(z=i \omega)$ is a constant in the whole frequency domain, whereas the imaginary one equals to zero, the expression (10) takes into account the frequency dispersion of the memory function, which will be discussed a bit later. We shall refer to the relation (11) as the Markovian approximation at the $s$-th hierarchy level (MAs), introducing an extra symbol $s$ in the above mentioned abbreviation. In the subsequent Sections, we consider the kinetic kernels for a particular number of $s=2 \div 5$, or, what is the same, use the approximations $(\mathrm{MA} 2) \div($ MA5). Hereafter, the index $s$, which denotes the chosen approximation level and is related to the highest order kinetic kernel, should not be confused with the "current" indexes $j \leq s$.

In particular, it would be instructive to compare the result for the VAF in the MA2 approximation with that obtained within the GCM theory ${ }^{8}$. To this end, let us use the recurrence relation (3) along with the MA for the highest order memory kernel:

$$
\tilde{\phi}_{s-1}(z) \approx \frac{\Gamma_{s-1}}{z+\tilde{\phi}_{s}(0)}=\frac{\Gamma_{s-1}}{z+\sqrt{\Gamma_{s}}} .
$$

Taking $s=2$ in Eq. (12) and relating the SCFs $\Gamma_{1}, \Gamma_{2}$ to the self-diffusion coefficient $D=1 / 3 \int_{0}^{\infty} d t\left\langle\mathbf{v}_{i}(t) \mathbf{v}_{i}(0)\right\rangle$ by the equation $\frac{\sqrt{\Gamma_{2}}}{\Gamma_{1}}=\frac{m D}{k_{B} T}$, we can reproduce the two-modes

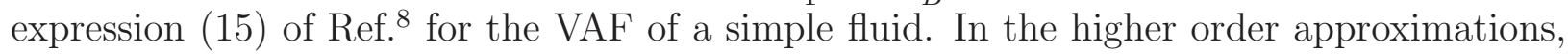
this result can be generalized as

$$
\frac{m D}{k_{B} T}=\left\{\begin{array}{l}
\frac{\Gamma_{2} \cdot \Gamma_{4} \cdots \Gamma_{2 k}}{\Gamma_{1} \cdot \Gamma_{3} \cdots \Gamma_{2 k-1}} \frac{1}{\sqrt{\Gamma_{2 k+1}}}, s=2 k+1 \\
\frac{\Gamma_{2} \cdot \Gamma_{4} \cdots \Gamma_{2 k-2}}{\Gamma_{1} \cdot \Gamma_{3} \cdots \Gamma_{2 k-1}} \quad \sqrt{\Gamma_{2 k}}, \quad s=2 k,
\end{array}\right.
$$

allowing one to compare the results obtained within the MAs with those calculated in the framework of the GCM s-modes approximation. However, as we have already mentioned, this equivalence with the GCM is mainly from a mathematical viewpoint, since in the GCM the self-diffusion coefficient is determined via a zero-time moment of the VAF, being calculated by the computer simulations. In the MAs, we use the SCFs $\Gamma_{j}$ as the input parameters (which also should be evaluated in computer simulations) and do not concern in the correlation time $\tau_{d i f}=m D / k_{B} T$, related to the self-diffusion.

It is worthy to note that the real part, $\operatorname{Re}\left[\tilde{\phi}_{s-1}(i \omega)\right]$, of the Lorenz-type kinetic kernel (12) decays as $\omega^{-2}$ in the high frequency domain, whereas the imaginary part decays as $\omega^{-1}$. In time representation, it corresponds to the exponential relaxation. In its turn, the real 
part of the highest order kinetic kernel $\tilde{\phi}_{s}(i \omega)$, defined by Eq. (10), sharply vanishes at the cut-off frequency $\omega_{c}=2 \sqrt{\Gamma_{s}}$, while its imaginary part after the lapse of the linear growth decays as $\omega^{-1}$ at large frequencies. It can be easily shown using the recurrence relation (3) that the same is true for the highest but one order memory kernel,

$$
\tilde{\phi}_{s-1}(i \omega)=\frac{\Gamma_{s-1}}{\Gamma_{s}} \tilde{\phi}_{s}(i \omega)
$$

Moreover, these results look even more interesting, if one performs the inverse Laplace transformation to (10),

$$
\phi_{s}(t) \equiv \frac{1}{2 \pi i} \lim _{\epsilon \rightarrow 0} \int_{\epsilon-i \infty}^{\epsilon+i \infty} e^{z t} \tilde{\phi}_{s}(z) d z=\sqrt{\Gamma_{s}} \frac{J_{1}\left(2 \sqrt{\Gamma_{s}} t\right)}{t},
$$

where the Bessel function of the first order $J_{1}$ appears. It means that the $s$-th and the $(s-1)$ th order memory kernels decay non-monotonically at long times as $t^{-3 / 2}$, since the Bessel function $J_{1}$ has the well known asymptote $J_{1}\left(2 \sqrt{\Gamma_{s}} t \gg 1\right)=\cos \left(2 \sqrt{\Gamma_{s}}(t-3 \pi / 4)\right) / \sqrt{\pi t}$. Thus, their dynamics completely differs from the time behavior of the highest order memory functions in the MA, which are $\delta$-correlated in time, and from the $(s-1)$-th order kinetic kernels in the MA, which are found to have an exponential relaxation, see Eq. (12).

Eqs. (10) and (15), obtained in the framework of the rigorous approach using just one physically reasonable assumption (9), are the cornerstones for our further study of the VAFs dynamics. From the mathematical point of view, we have done nothing but an effective summation of the infinite continued fraction (5), in which all the parameters $\Gamma_{j}$ are equal to each other starting from the certain value $j=s$. Thus, in fact, we deal with the problem with a limited number $s$ of the parameters like it is within the GCM (MA) approaches. In the next Section we consider the spectral functions with various values of $s$, at which the condition (9) are supposed to hold true.

\section{SPECTRAL FUNCTIONS}

Let's consider the spectral function $(\mathrm{SF}) \tilde{F}_{v}(\omega)=\operatorname{Re}\left[\tilde{F}_{v}(z=i \omega)\right]$, defined as a real part of the corresponding continued fraction (5) taken at the imaginary frequency. In this Section, we replace the label $v$ in the expressions for SFs by the subscript $\alpha$, which denotes the chosen level of approximation (see below for details). 
At the beginning, let us adopt the approximation of the second hierarchy level. In such a case, it is straightforward to evaluate the $\mathrm{SF} \tilde{F}_{2}(\omega)$,

$$
\tilde{F}_{2}(\omega)=\frac{\Gamma_{1} \sqrt{\Gamma_{2}-\omega^{2} / 4}}{\left(\Gamma_{2}-\Gamma_{1}\right) \omega^{2}+\Gamma_{1}^{2}} .
$$

It is also instructive to compare the result (16) with the expression

$$
\tilde{F}_{M 2}(\omega)=\frac{\Gamma_{1} \sqrt{\Gamma_{2}}}{\omega^{4}+\left(\Gamma_{2}-2 \Gamma_{1}\right) \omega^{2}+\Gamma_{1}^{2}}
$$

for the SF, obtained by a truncation of the continued fraction at the level $s=2$. Hereafter, we use the extra subscript $M$ to denote the above mentioned MA (or modes) approximation.

It is useful to compare the expressions (16)-(17) for the corresponding SFs. First of all, the former spectral function vanishes at the cut-off frequency $\omega_{c}=2 \sqrt{\Gamma_{2}}$, while the latter tends to zero asymptotically as $1 / \omega^{4}$.

Secondly, both SFs are even functions of frequency and behave as $\lim _{\omega \rightarrow 0} d \tilde{F}_{\alpha}(\omega) / d \omega=0$, here $\alpha$ denotes 2 or M2. Thirdly, in the domain of frequencies $\omega \ll \omega_{c}$ and at $\Gamma_{1} \ll$ $\Gamma_{2}$, the relation $\tilde{F}_{2}(\omega) \approx \tilde{F}_{M 2}(\omega)$ holds true. It means that at a certain relation between the parameters $\Gamma_{j}$, the results, obtained within our approximation and the GCM (MA) formalisms, are close to each other (we will touch upon this issue in more detail in Sec. V, when analyzing the time behavior of the corresponding VAFs).

At last but not least, in the vicinity $\varepsilon$ of the cut-off frequency $\omega_{c}$ the SF (16) behaves as

$$
\tilde{F}_{2}\left(\omega_{c}-\varepsilon\right) \approx \frac{\Gamma_{1} \Gamma_{2}^{1 / 4}}{\left(\Gamma_{1}-2 \Gamma_{2}\right)^{2}} \sqrt{\varepsilon}
$$

The square root dependence (18) resembles the well-known result from the MCT 20,21 . However, in the MCT approach a non-analytical dependence $\sqrt{\omega}$ of the SFs, generating the long-time tails $\sim t^{-3 / 2}$ of the VAFs, occurs at the zero frequency domain rather than at $\omega_{c}$.

A similar structure of the SF can be obtained at the approximation of the third hierarchy level. In this case, the spectral function $\tilde{F}_{3}(\omega)$, obtained by summing up the continued fraction for the 3-rd order memory kernel, can be presented as follows:

$$
\tilde{F}_{3}(\omega)=\frac{\Gamma_{1} \Gamma_{2} \sqrt{\Gamma_{3}-\omega^{2} / 4}}{\left(\Gamma_{3}-\Gamma_{2}\right) \omega^{4}+\left(\Gamma_{2}\left(\Gamma_{1}+\Gamma_{2}\right)-2 \Gamma_{1} \Gamma_{3}\right) \omega^{2}+\Gamma_{1}^{2} \Gamma_{3}},
$$

while its counterpart in the modes approximation looks as

$$
\tilde{F}_{M 3}(\omega)=\frac{\Gamma_{1} \Gamma_{2} \sqrt{\Gamma_{3}}}{\omega^{6}-\left[2\left(\Gamma_{1}+\Gamma_{2}\right)-\Gamma_{3}\right] \omega^{4}+\left[\left(\Gamma_{1}+\Gamma_{2}\right)^{2}-2 \Gamma_{1} \Gamma_{3}\right] \omega^{2}+\Gamma_{1}^{2} \Gamma_{3}} .
$$


Once again, like in the the cases (16)-(17), two SFs are close to each other, $\tilde{F}_{3}(\omega) \approx$ $\tilde{F}_{M 3}(\omega)$ in the domain of frequencies $\omega \ll \omega_{c}$ and at $\Gamma_{3} \gg \max \left\{\Gamma_{1}, \Gamma_{2}\right\}$.

Inspecting Eq. (16) and taking into account the sum rule

$$
\frac{1}{2 \pi} \int_{-\infty}^{\infty} \tilde{F}_{s}(\omega) d \omega \equiv \frac{1}{2 \pi} \int_{-\omega_{c}}^{\omega_{c}} \tilde{F}_{s}(\omega) d \omega=1
$$

(which, in fact, denotes the initial value of the VAF being equal to the unity), one can observe by a direct integration that there is a minimal value $\Gamma_{2}^{(\text {min })}=\Gamma_{1} / 2$, below which the condition Eq. (21) is no longer valid.

In a similar way, the value $\Gamma_{3}^{(m i n)}=\left(\Gamma_{1}+2 \Gamma_{2}\right) / 4$ can be defined for the fixed (lower order) SCFs $\Gamma_{1}, \Gamma_{2}$ in Eq. (19), and the sum rule (21) is satisfied for $\Gamma_{3} \geq \Gamma_{3}^{(\min )}$. In this context, our results differ from those (see Eqs. (17), (20)), obtained within the modes approximation: in the latter case, no relation between various $\Gamma_{i}$ is needed for the sum rule (21) to be satisfied.

We have to emphasize that so far the minimal values $\Gamma_{s}^{(m i n)}$ appear in a strictly "mathematical" way due to the requirement (21). In a recent paper Ref. 31 , we have attributed more physical meaning for $\Gamma_{s}^{(\min )}$. This parameter was shown to be the threshold value, which defines a crossover from the power law $\sim t^{-3 / 2}$ to the slower relaxation $\sim t^{-1 / 2}$ of the VAF, when the fluid starts to behave like a typical 1D-system ${ }^{38}$. However, computer simulation data for the fluids show (see Sec. IV) that the highest order SCFs are well above their minimally allowed values $\Gamma_{s}^{(\min )}$. Thus, a possible solid-like scenario of the fluid dynamics, which has been discussed in Ref. $\underline{31}$, is not realized in our case.

It is straightforward to obtain the SFs similar to (16)-(17), (19)-(20) at any value of the hierarchy order $s$. Like in the above presented case, the highest order $\mathrm{SCF} \Gamma_{s}$ would determine the corresponding cut-off frequency $\omega_{c}=2 \sqrt{\Gamma}_{s}$. The frequency expansion of the obtained SFs in the vicinity of $\omega_{c}$ would also start from the square root term (see Eq. (18)) with the renormalized coefficient due to taking into account of the higher order $\Gamma_{s}$.

The above mentioned functions can be the starting points that allow us to study a time behavior of the corresponding VAFs, using the values for $\Gamma_{j}, j=\{1, \ldots, s\}$, taken from the MD simulations. The computer simulation data as well as the results of the theoretical prediction are presented in the next two Sections. 


\section{TIME BEHAVIOR OF THE AUTOCORRELATION FUNCTIONS: COMPUTER SIMULATIONS}

In our recent paper $\underline{\underline{31}}$ we have calculated the VAFs at various values of $\Gamma_{j}$, regardless of their relations to the fluid at a particular thermodynamic point. We have presented different regimes of the fluids dynamics (when the system behaves in a solid-like, liquid-like or gas-like manner), modelled in the framework of such an approach. Being aware that some (initially prescribed) combinations of $\Gamma_{j}$ can be unrepresentative for the real fluids, in this Section we study the dynamics of $\mathrm{Ne}$ at some particular thermodynamic points.
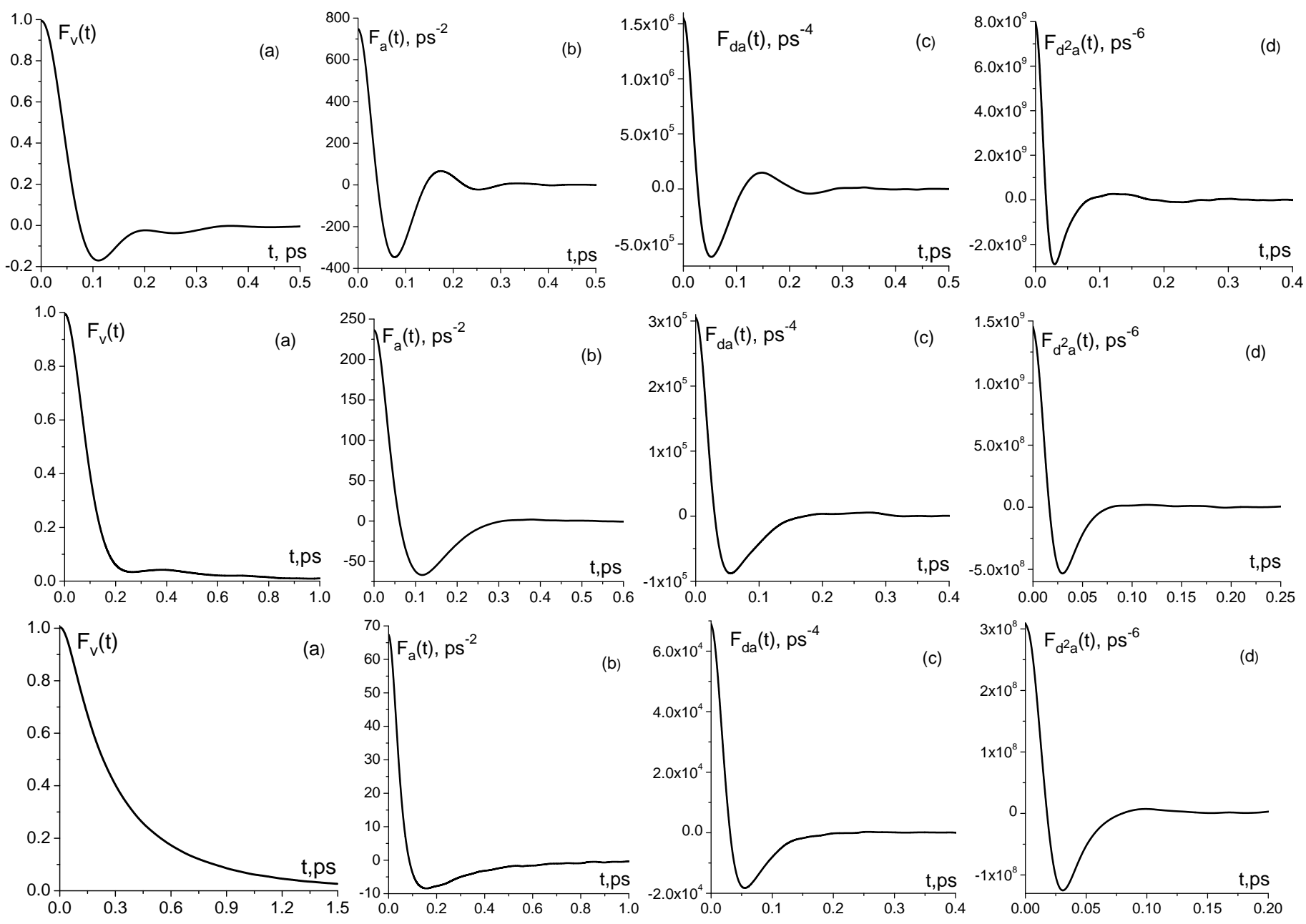

FIG. 1. Time correlation functions $F_{v}(t)=\left\langle\mathbf{v}_{i} \mathbf{v}_{i}(t)\right\rangle /\left\langle\mathbf{v}_{i} \mathbf{v}_{i}\right\rangle$ (a), $F_{a}(t)=\left\langle\mathbf{a}_{i} \mathbf{a}_{i}(t)\right\rangle /\left\langle\mathbf{v}_{i} \mathbf{v}_{i}\right\rangle$ (b), $F_{d a}(t)=\left\langle\dot{\mathbf{a}}_{i} \dot{\mathbf{a}}_{i}(t)\right\rangle /\left\langle\mathbf{v}_{i} \mathbf{v}_{i}\right\rangle$ (c) and $F_{d^{2} a}(t)=\left\langle\ddot{\mathbf{a}}_{i} \ddot{\mathbf{a}}_{i}(t)\right\rangle /\left\langle\mathbf{v}_{i} \mathbf{v}_{i}\right\rangle$ (d) of Ne taken at the temperature $T=295 \mathrm{~K}$ and densities $\rho=2190 \mathrm{~kg} / \mathrm{m}^{3}$ (upper row), $\rho=1400 \mathrm{~kg} / \mathrm{m}^{3}$ (middle row) and $\rho=691$ $\mathrm{kg} / \mathrm{m}^{3}$ (lower row). 
Molecular dynamics simulations were performed on a system of 4000 particles interacting via pair Lennard-Jones potential with parameters corresponding to Ne. The simulation setup and effective potentials were essentially the same as in the previous study on supercritical $\mathrm{Ne}^{39}$. We performed simulations for 12 densities in the range from $691 \mathrm{~kg} / \mathrm{m}^{3}$ to $2190 \mathrm{~kg} / \mathrm{m}^{3}$ of supercritical Ne at temperature $295 \mathrm{~K}$ with the purpose to estimate time evolution and calculate autocorrelations of particle velocities, accelerations and their higher time derivatives. The time step in simulations in the microcanonical ensemble was $0.5 \mathrm{fs}$, and the pressure calculated from MD simulations was in perfect agreement with the available NIST data $\frac{40}{}$ for densities of supercritical Ne up to $1200 \mathrm{~kg} / \mathrm{m}^{3}$. The analytical expressions for the acceleration and its derivatives,

$$
\begin{aligned}
& \mathbf{a}_{i}=\dot{\mathbf{v}}_{i}=\sum_{j \neq i=1}^{N} \mathbf{a}_{i j}, \quad \mathbf{a}_{i j}=-\frac{1}{m} \Phi^{\prime}\left(r_{i j}\right) \frac{\mathbf{r}_{i j}}{r_{i j}}, \quad \Phi^{\prime}(r)=\frac{d \Phi(r)}{d r}, \\
& \dot{\mathbf{a}}_{i}=\frac{1}{m} \sum_{j \neq i=1}^{N}\left\{-\Phi^{\prime}\left(r_{i j}\right) \frac{\mathbf{v}_{i j}}{r_{i j}}-\left[r_{i j} \Phi^{\prime \prime}\left(r_{i j}\right)-\Phi^{\prime}\left(r_{i j}\right)\right] \frac{\mathbf{r}_{i j}\left(\mathbf{r}_{i j} \cdot \mathbf{v}_{i j}\right)}{r_{i j}^{3}}\right\}, \\
& \ddot{\mathbf{a}}_{i}=-\frac{1}{m} \sum_{j \neq i=1}^{N}\left\{\Phi^{\prime}\left(r_{i j}\right) \frac{\mathbf{a}_{i j}}{r_{i j}}+\left[r_{i j} \Phi^{\prime \prime}\left(r_{i j}\right)-\Phi^{\prime}\left(r_{i j}\right)\right]\right. \\
& \left.\times\left[\frac{2 \mathbf{v}_{i j}\left(\mathbf{r}_{i j} \cdot \mathbf{v}_{i j}\right)+\mathbf{r}_{i j}\left(\mathbf{v}_{i j}^{2}+\left(\mathbf{r}_{i j} \cdot \mathbf{a}_{i j}\right)\right)}{r_{i j}^{3}}-\frac{3 \mathbf{r}_{i j}\left(\mathbf{r}_{i j} \cdot \mathbf{v}_{i j}\right)^{2}}{r_{i j}^{5}}+\Phi^{\prime \prime \prime}\left(r_{i j}\right) \frac{\mathbf{r}_{i j}\left(\mathbf{r}_{i j} \cdot \mathbf{v}_{i j}\right)^{2}}{r_{i j}^{3}}\right]\right\}, \\
& \mathbf{r}_{i j}=\mathbf{r}_{i}-\mathbf{r}_{j}, \quad \mathbf{v}_{i j}=\mathbf{v}_{i}-\mathbf{v}_{j}, \quad \mathbf{a}_{i j}=\mathbf{a}_{i}-\mathbf{a}_{j},
\end{aligned}
$$

involving the derivatives of Lennard-Jones potentials $\Phi(r) \equiv \Phi_{L J}(r)$, were used to estimate their time evolution along particle trajectories in MD simulations.

To investigate the system dynamics in more detail, we do not limit ourselves by the VAFs only and perform computer simulations for the acceleration autocorrelation functions as well as for the TCFs, defined on the higher derivatives of the velocity up to the 4-th order. Except for a purely theoretical interest, the obtained results have an applied aspect too, providing us with the values $\Gamma_{j}$ that are used in our method.

The results of MD simulations for some of the TCF of Ne are presented in Fig. 1. It is seen that the VAF of the fluid at the highest studied density $\rho=2190 \mathrm{~kg} / \mathrm{m}^{3}$ has a pronounced minimum at $t=0.1 \mathrm{ps}$. This minimum is related to the back-scattering of the fluid particle trapped in the cage, which is formed by its neighborhood. At the intermediate times, vibration of the particle causes a rearrangement of the solvation shell, allowing the particle to travel away from its initial position, until it is retrapped at $t=0.25$ ps by another cage 
with much less local ordering of the particles. At larger times, the particle escapes from all local traps, and the oscillations become completely damped.

At the intermediate density $\rho=1400 \mathrm{~kg} / \mathrm{m}^{3}$, the effects of the local ordering of the molecules are almost negligible except the time $t=0.3 \mathrm{ps}$, where the corresponding VAF has an inflection point. At the density $\rho=691 \mathrm{~kg} / \mathrm{m}^{3}$, the molecules packing is too small to produce any sign of the local ordering, which would be able to trap the particle, and the VAF smoothly decays on the whole time domain.

The results of particle dynamics in terms of the VAFs are interesting to compare with those of acceleration autocorrelation functions $F_{a}(t)$, depicted in the column b) of Fig. 1. At the density $\rho=2190 \mathrm{~kg} / \mathrm{m}^{3}$, this function changes its sign in the time interval $0.04 \div 0.14 \mathrm{ps}$. It means that the force, acting on the Ne particle, reverses its direction due to the particle scattering on surrounding molecules. Thus, the interval about 0.05 ps can be associated with the effective time between collisions.

On the other hand, the depth of the well in the acceleration autocorrelation function strongly decreases with the density reduction. This phenomenon can be explained in the following way: at low densities the particle changes its direction of motion due to the multiple low-angle scattering whereas at high density the "head-on" collisions dominate, yielding the profound minimum in $F_{a}(t)$.

The last two columns in Fig. 1 show that autocorrelation functions, associated with higher derivatives of force, decay much faster than $F_{a}(t)$, while locations of the minima cease to depend on the system density. It is quite expected, since the rate of the force change is defined by a continuous interaction of the tagged particle with its surrounding rather than by an effective (density dependent) collision mechanism between the molecules. The same is also true for the autocorrelation functions $F_{d^{2} a}(t)$, while their relaxation times are shorter as compared with those of $F_{a}(t)$, since the former TCFs are constructed on much faster dynamical variables. The autocorrelation functions, dealt with the third derivative of the force, bring no essential physics to understanding of the fluid dynamics, and, consequently, are not presented in Fig. 1.

The evaluated autocorrelation functions allow us to obtain the values of $\Gamma_{s}$, which are the input parameters in our theory. Indeed, zero time values of the autocorrelation functions $F_{a}(t), F_{d a}(t), F_{d^{2} a}(t)$ define, correspondingly, the 2-nd, the 4-th and the 6-th frequency 
moments of the VAF,

$$
\left\langle\omega^{2}\right\rangle=\frac{\left\langle\mathbf{a}_{i} \mathbf{a}_{i}\right\rangle}{\left\langle\mathbf{v}_{i} \mathbf{v}_{i}\right\rangle}, \quad\left\langle\omega^{4}\right\rangle=\frac{\left\langle\dot{\mathbf{a}}_{i} \dot{\mathbf{a}}_{i}\right\rangle}{\left\langle\mathbf{v}_{i} \mathbf{v}_{i}\right\rangle}, \quad\left\langle\omega^{6}\right\rangle=\frac{\left\langle\ddot{\mathbf{a}}_{i} \ddot{\mathbf{a}}_{i}\right\rangle}{\left\langle\mathbf{v}_{i} \mathbf{v}_{i}\right\rangle},
$$

which can be calculated directly via the positions $\mathbf{r}_{i}$ and velocities $\mathbf{v}_{i}$ of all the particles in computer simulations using the microscopical equations of motion (22). The expressions for higher order frequency moments can be obtained straightforwardly. In our study, we calculate frequency moments up to the 8-th order directly by MD, and only the 10-th order frequency moments are evaluated approximately by taking the second order derivative of the autocorrelation functions $\left\langle\dddot{\mathbf{a}}_{i} \dddot{\mathbf{a}}_{i}(t)\right\rangle /\left\langle\mathbf{v}_{i} \mathbf{v}_{i}\right\rangle$ at $t=0$.

The above mentioned frequency moments can be connected by the relations

$$
\begin{gathered}
\Gamma_{1}=\left\langle\omega^{2}\right\rangle, \quad \Gamma_{2}=\frac{\left\langle\omega^{4}\right\rangle}{\left\langle\omega^{2}\right\rangle}-\left\langle\omega^{2}\right\rangle, \quad \Gamma_{3}=\frac{\left\langle\omega^{6}\right\rangle\left\langle\omega^{2}\right\rangle-\left\langle\omega^{4}\right\rangle^{2}}{\left\langle\omega^{4}\right\rangle\left\langle\omega^{2}\right\rangle-\left\langle\omega^{2}\right\rangle^{3}}, \\
\Gamma_{4}=\frac{1}{\Gamma_{1} \Gamma_{2} \Gamma_{3}}\left\{\left\langle\omega^{8}\right\rangle-\Gamma_{1}\left[\left(\Gamma_{1}+\Gamma_{2}\right)^{3}+2 \Gamma_{2} \Gamma_{3}\left(\Gamma_{1}+\Gamma_{2}\right)+\Gamma_{2} \Gamma_{3}^{2}\right]\right\}
\end{gathered}
$$

with the SCFs $\Gamma_{j}$, constructed on the orthogonal set of the dynamic variables. The expression for the SCFs $\Gamma_{j}$ with higher indexes $j$ can be evaluated straightforwardly using certain recurrent relations (see, for instance, Eq. (4) of Ref. 11 ).

\section{TIME BEHAVIOR OF THE VAFS: THEORY}

Expressions like (16)-(17) and (19)-(20) allow us to study the frequency dependence of SFs in the broad domain of parameters $\Gamma_{j}$ as it has been discussed in Sec. III. It has been shown in Ref 31 that the VAFs, obtained within our approach, have the same frequency moments up to the order $(2 s-2)$ as their counterparts, obtained within the $\mathrm{MA}^{7} \underline{7} \underline{\underline{*}}$. What is the most essential, our approach reproduces also the exact result for the next frequency moment, $\left\langle\omega^{2 s}\right\rangle=\frac{1}{2 \pi} \int_{-\omega_{c}}^{\omega_{c}} \omega^{2 s} \tilde{F}_{s}(\omega) d \omega$, that can be easily verified, for instance, by a direct integration of the corresponding SFs using Eqs. (16)-(17) and (19)-(20). In other words, we have an extra (correct) term $\sim t^{2 s}$ in the Taylor expansion series of the VAFs as compared with the MA case at the same number of the input parameters $\Gamma_{j}$. This allows us to consider the method based on the effective summation of the infinite continued fractions as more efficient at the description of the VAFs behavior not only at the small but also at the intermediate times. Besides, the square-root dependence (18) of the SFs causes the long-time tails appearance, though in a different manner as it is usually predicted in the $\mathrm{MCT} \underline{18} \underline{-20}$. 
To start with an investigation of the VAFs dynamics, we present in Table I the values of the lowest order SCFs $\Gamma_{1}$ and ratios $\Gamma_{j+1} / \Gamma_{j}$ at various densities. It is evident from the Table I that the above mentioned ratio tends noticeably to the unity with the increase of the hierarchy level $j$. Hence, our basic assumption about the fast convergence of the SCFs $\Gamma_{j}$ looks well-grounded.

TABLE I. Values of the SCFs $\Gamma_{1}$ and ratios $\Gamma_{j+1} / \Gamma_{j}$ at the highest, intermediate and lowest densities studied.

\begin{tabular}{lccccc}
\hline \hline & $\Gamma_{1}, \mathrm{ps}^{-2}$ & $\Gamma_{2} / \Gamma_{1}$ & $\Gamma_{3} / \Gamma_{2}$ & $\Gamma_{4} / \Gamma_{3}$ & $\Gamma_{5} / \Gamma_{4}$ \\
\hline$\rho=2190 \mathrm{~kg} / \mathrm{m}^{3}$ & 747.9 & 1.774 & 3.606 & 2.406 & 1.45 \\
$\rho=1400 \mathrm{~kg} / \mathrm{m}^{3}$ & 233.611 & 4.531 & 3.779 & 2.473 & 1.9 \\
$\rho=691 \mathrm{~kg} / \mathrm{m}^{3}$ & 67.385 & 14.377 & 3.843 & 2.521 & 1.665 \\
\hline \hline
\end{tabular}

A similar convergence tendency can be traced for the lowest order relaxation time $\tau_{1}=$ $1 / \tilde{\phi}_{1}(0)$, evaluated at various indexes $s=1 \div 5$ of the hierarchy level as it is clearly visible from Fig. 2. It is seen that $\tau_{1}$ oscillate around their values attainable at some large $s \gg 1$,

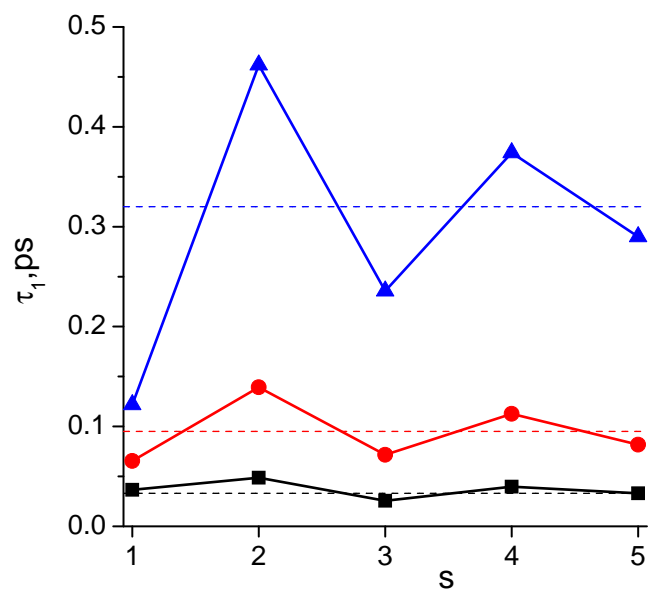

FIG. 2. Dependence of the lowest order relaxation time $\tau_{1}$ on the hierarchy level index $s$ at the fluid densities $\rho=691 \mathrm{~kg} / \mathrm{m}^{3}$ (triangles), $1400 \mathrm{~kg} / \mathrm{m}^{3}$ (circles), and $2190 \mathrm{~kg} / \mathrm{m}^{3}$ (squares). The dashed lines correspond to the values taken from the computer simulations. The straight lines are a guide for eye.

approaching them from above (even $s$ ) or below (odd $s$ ). A convergence grows rapidly with 
the density increase. Besides, the values of $\tau_{1}$ decrease with density, since the relaxation times of the memory kernels are lower in the dense fluids.
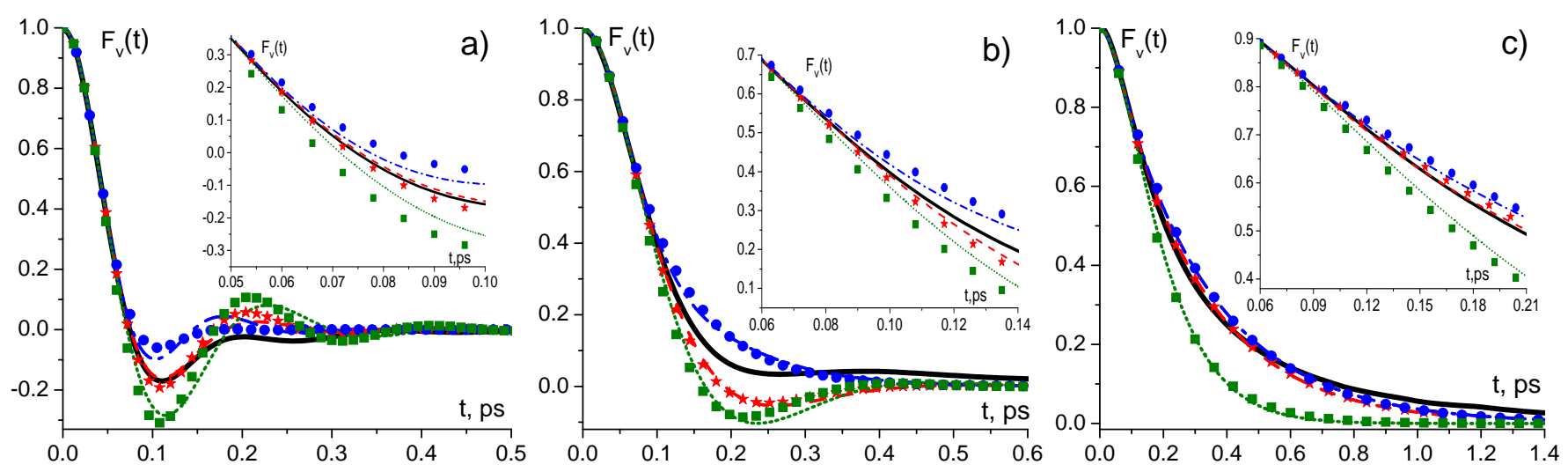

FIG. 3. VAFs of the liquid Ne at the densities $\rho=2190 \mathrm{~kg} / \mathrm{m}^{3}$ (a), $1400 \mathrm{~kg} / \mathrm{m}^{3}$ (b) and $691 \mathrm{~kg} / \mathrm{m}^{3}$ (c). The solid lines correspond to the MD data. Other lines correspond to the VAFs, obtained by an effective summation of the infinite continued fractions up to the 3-rd (dotted line), 4-th (dashed-dotted line) and 5-th (dashed line) hierarchy levels. The symbols correspond to the VAFs, obtained within MA3 (squares), MA4 (bullets) and MA5 (stars) approximations. In the insets: time behavior of the corresponding VAFs at small $t$.

Thus, having the values of all the input parameters $\Gamma_{j}, j=\{1, \ldots, 5\}$, and performing the inverse Fourier transformation of the corresponding SFs, which can be obtained in a similar way as those in Sec. III, we are able to calculate the velocity autocorrelation functions at different levels of approximation. The results for the VAFs of liquid Ne at various densities, calculated directly by MD simulations, as well as those obtained analytically at different approaches are presented in Fig. 3.

One can draw several conclusions when inspecting Fig. 3. First of all, a convergence of all results to the MD data (denoted by the solid master curve) with increasing of the approximation order is clearly noticeable. Both lines (corresponding to our approach) and symbols (related to the modes approximation) follow the solid black line the better, the higher $\Gamma_{s}$ are taken into account. It is quite expected, since by increasing the hierarchy level $s$ we ensure more frequency moments of the VAFs to be satisfied.

Secondly, as we have mentioned at the beginning of this Section, the effective summation of the infinite continued fractions ensures more terms in time series of the VAFs that reproduce the exact fluid dynamics as compared with the fractions truncation (which has 
been shown to be equivalent to the mode approximation). Indeed, it is seen in the insets of Fig. 3 that for all the densities the curves, corresponding to the fixed level of approximation $s$, leave the solid master curve later than their symbolic counterparts.

Thirdly, and what is the most surprising, the dashed curve at the highest reported density coincides with the solid one in a rather broad time interval, including that of the minimum of VAF, related to the cage effect. Moreover, the difference between the results, obtained in our approach (lines), and those, obtained by a truncation of the continued fractions (symbols), is quite noticeable. At the lowest density, this difference almost vanishes, and a question, which method should be chosen to describe the fluid dynamics, becomes less relevant.

Now one remark is to the point. It is possible to calculate the generalized memory kernels of different orders using the MD data and compare them with those, evaluated by an effective summation of the continued fractions or by the MA. The point is that the autocorrelation functions of different orders obey the recurrence relations $\frac{11}{}$, allowing one to express the generalized kinetic kernels $\tilde{\phi}_{j}(z)$ via SCFs and Laplace transforms of the TCFs (see, for instance, Eqs. (6)-(9b) of the cited paper). However, to evaluate the dynamics of kernels at the same accuracy level as it has been done in the case of VAFs (Fig.3), one has to possess (at least) the same number of SCFs $\Gamma_{j}$, which should be of higher orders as compared with those used in our study. In particular, to calculate reliably the 1-st order kernel we need the 6 -th order SCF and so on. Since, in any case, we have a limited number of parameters, obtained from the MD calculations, a dynamics of the memory functions $\tilde{\phi}_{j}(z)$ (even started from $j=1$ ) would be obtained with worse accuracy than that of VAFs.

As we have already mentioned, the long time behavior of the TCFs remains a topical problem in the physics of fluids $\underline{18}-21,30$. In our recent paper $\underline{31}$, we have paid much attention to the studies of the long-time tails formation in fluids. In particular, we obtained quite simple expressions for the transition time $\tau_{H}$ to the hydrodynamic regime at the assumption that all the $\Gamma$ converge to their asymptotic value starting from $s=2$. We have shown that there could be not only the simple poles of the corresponding SFs, but also another kind of discontinuity, like the essential singularity points. Since the kinds of the singularities and the localization of the discontinuities on the complex frequency plane are known to define 28,29 the values of $\tau_{H}$, this fact brings new features into the long time dynamics of the VAFs, renormalizing the corresponding transition times.

A similar attempt to express the transition times analytically can be performed at the 
approximation of the $s$-th hierarchy level. However, it is much more instructive to estimate $\tau_{H}$ numerically at several thermodynamic points, taking the values of $\Gamma_{j}$, obtained by computer simulations, as the input parameters.

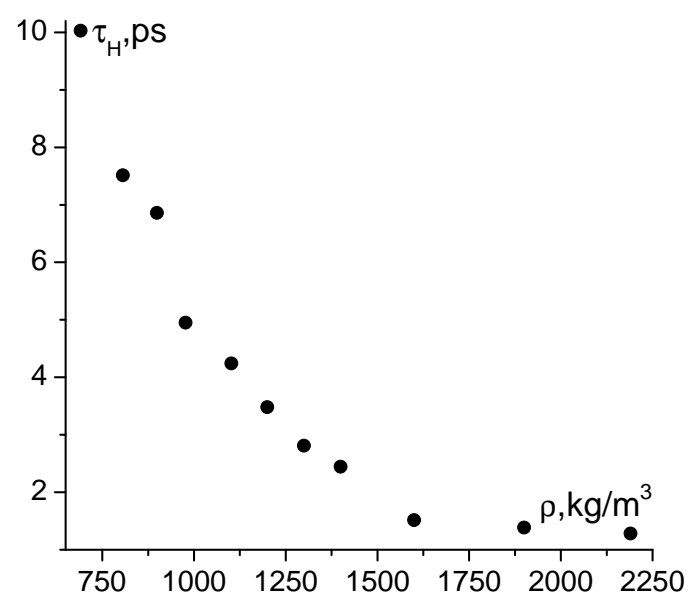

FIG. 4. Dependence of the transition time $\tau_{H}$ to the hydrodynamic regime on the fluid density.

In Fig. 4 we present the density dependence of the time $\tau_{H}$, needed for the fluid to reach the hydrodynamic stage of evolution. In our calculation, we have performed an effective summation of the infinite continued fraction within the approach, described in Sec. II, and used the corresponding SCFs up to the order $s=4$, for which the direct MD data are available. The transition time was estimated by the contact point of the envelope curve $a t^{-3 / 2}$ and the corresponding VAF.

It is seen from Fig. 4 that the transition time weakly depends on $\rho$ at the superdense states of the fluid. Then, starting from $\rho<1500 \mathrm{~kg} / \mathrm{m}^{3}$, it grows by almost an order of magnitude at the smallest density studied. If we recall a concept of the effective time between particle collisions, which can be related to the instant, when the "force-force" autocorrelation function changes its sign (see the column (b) of Fig. 1), it becomes evident, that from 30 (at the highest density) to about 100 (at the lowest density) particle collisions are required to form the long-time tails in the VAFs. In this context, our results agree with those, obtained in Ref. $\frac{12}{}$, where the number of particle collisions prior to the long-time tails appearance was estimated by the ratio of $\tau_{H}$ and the Enskog mean time $\tau_{E}$ between collisions for the equivalent hard spheres fluid. On the other hand, the transition time at $\rho \sim 1500 \mathrm{~kg} / \mathrm{m}^{3}$ and $T=295 \mathrm{~K}$ (that correspond to the dimensionless density $\rho \sigma_{L J}^{3}=1$ and temperature $k_{B} T / \varepsilon_{L J}=8$ ), being expressed in the dimensionless time units $\tau_{H} / \tau_{L J}$, 
$\tau_{L J}=\sigma_{L J} \sqrt{m / \varepsilon_{L J}}$, is close to the MD data of Ref.41 (look at the vicinity of the first bullet in the inset in Fig. 3(c) of the cited paper).

As we have already mentioned, our approach can be quite promising at the study of the crossover to the Navier-Stokes hydrodynamics, since it provides a description of the SFs topology in the complex frequency plane as well as a systematization of the singularities types 28,29 , whereas the MCT does not give a direct recipe for estimation of this value. However, in contrast to the MCT, the above presented scheme does not allow to calculate reliably, for instance, the long tail amplitude $a$. Anyway, we do not expect our results to agree quite satisfactory with those predicted by the MCT, since these two methods rest upon different basic points.

In our theory, we do not operate explicitly by any dissipative characteristics of the system, originating from the hydrodynamically based MCT approach, like the viscosity or self-diffusion coefficient (though the last one can be expressed by the corresponding spectral functions taken at zero frequency, see Sec. III). The fluid dynamics is completely defined by the static characteristics of the system given by the parameters $\Gamma_{j}$, and the obtained results well agree with the computer simulations data in the quite broad time domain of the system evolution.

In the framework of MCT, a basic set of the dynamic variables consists of higher $\operatorname{products}^{20}$ rather than higher derivatives of the densities of conserved quantities. In the MCT approach $\underline{18}, 20$, the system dynamics is well described at the long times, but this method does not allow to obtain the reliable results at other timescales (in particular, the results obtained within MCT based methods do not obey the sum rules).

To conclude the discussion about an interrelation of our method and the MCT, we would like to mention some important points. To a certain extent, our theoretical scheme can formalize the idea of a self-consistent description of the density fluctuations in a similar manner as this is done by the MCT, where the description of the system dynamics is made by the closed integro-differential equations ${ }^{42}$. Indeed, Eqs. (44) are of the similar structure as those considered in the framework of MCT: we can treat the relation (14) as an ansatz which allows to express the highest order memory function via the $(s-1)$-th order kernel to close the chain of equations.

What is more interesting, the above mentioned formal similarity between our method and the MCT does not end at this point. There is one more common feature of both 
approaches: a non-analytic dependence of the SFs on frequency. However, in the MCT framework it occurs in the zero frequency domain $\stackrel{21}{ }$, while in our approach the corresponding SFs depend non-analytically on $\omega$ in the vicinity of the cut-off frequency $\omega_{c}$, see Eq. (18). The coefficient at the square root in (18) defines the value of the long tail amplitude at the accepted condition of the 2-nd order hierarchy.

However, as we have already mentioned, this value would differ from the prediction of the MCT. Moreover, one should speak about a certain envelope curve rather than the long-time tail $a t^{-3 / 2}$. The point is that our assumption about very rapid (basically, instantaneous) convergence of the SCFs $\Gamma_{j}$ to their asymptotic limit is rather oversimplified. Actually, one deals with a much slower convergence of the higher order relaxation times with $j$. The above mentioned oversimplification leads to a pronounced modulation of the obtained long-time tails, as discussed in detail in our recent paper ${ }^{31}$.

One of the possible ways to diminish these oscillations consists in a construction of some kinds of the periodic continued fractions according to the following ansatz:

$$
\tilde{\phi}_{s}(z) \approx \tilde{\phi}_{s+n}(z), \quad n \geq 2
$$

From the physical point of view, it corresponds to a more realistic approximation for the VAFs, when the high order relaxation times approach their asymptotic values, alternately, from below or above ${ }^{37}$ rather than instantly, as it happens within our scheme.

From the mathematical viewpoint, we obtain a bilinear form with respect to the highest order memory kernel $\tilde{\phi}_{s}(z)$, like it was in the case of our basic approximation (9). However, a discriminant of the quadratic equation would have a much more complicated structure, being the $2 n$-th order polynomial in $\omega$. Zeros of the above mentioned polynomial define the cut-off frequencies $\omega_{c i}, i=\{1, \ldots, n\}$ and, consequently, the gaps in the SFs. With increasing of $n$, the interval of the frequencies $\left[\omega_{c 1}, \omega_{c n}\right]$ broadens; at the same time, the lowest cut-off frequency $\omega_{c 1}$ moves to the left, and the highest cut-off frequency $\omega_{c n}$ shifts to the right. The frequency behavior of the SFs at the gaps' edges is very similar to (18), being proportional to $\sqrt{\left|\omega-\omega_{c i}\right|}$. The coefficients of the proportionality (long tails amplitudes $a_{i}$ ) can be ordered by their magnitudes, $\left|a_{1}\right|>\left|a_{2}\right|>\ldots\left|a_{n}\right|$. Due to the low frequency shift of the $\omega_{c i}$ at small $i$ and to a negligible contribution of the terms with large $i$, the net effect would be a smoothing of the oscillations in the long-time tails of the VAFs. It is clear that a construction of such periodic continued fractions has to be well grounded from the physical 
viewpoint (in the first turn, due to requirement of the sum rules ${ }^{31}$ ), and could be a subject of the separate studies.

\section{CONCLUSIONS}

In this paper, we propose a simple ansatz for the high order kinetic kernels of the fluid that allows us to model the system dynamics on a quite broad time interval. Our approach is based on the physically grounded assumption about convergence of the relaxation times of the high order memory functions 31 ,37, which have a purely kinetic origin. As a result, the infinite continued fractions for the Laplace transforms of the VAFs can be effectively summed up, yielding the closed form that depend only on limited number of the SCFs, which can be taken from computer simulations.

To a certain extent, such an approach resembles the GCM formalism $\underline{\underline{5}-\underline{8}}$, which also assumes a description of the system by a finite set of the parameters (the static correlation functions and corresponding relaxation times). However, the distinctive feature of our approach is that, in contrast to the GCM theory (or interrelated with it the MA formalism), there is an intrinsic frequency dispersion in the memory kernels of all orders.

The above mentioned dispersion turns out to have a double impact on the fluid dynamics. First of all, it provides a larger number of terms in the time series of the VAFs that agree with the MD simulations data, allowing one to describe the system dynamics at short and

intermediate times more precisely as compared with the GCM (MA) at the same level $s$ of the hierarchy. Quite unexpectedly, our approach was found to be especially promising at the description of the super dense fluids, while at the lower densities the difference from the results, obtained within MA framework, becomes less evident, and the issue which method is more preferable becomes less relevant.

Secondly, unlike the GCM, an application of our method reaches far beyond this time domain, making it possible to estimate the transition times from the kinetic to the hydrodynamic stages of evolution, and even to study the processes of long-time tails formation (though, at least in its present form, our approach cannot compete with the MCT in the description of late stages of the system dynamics). In this context, our method has some common features with the so-called "combined" ones $\underline{28}, \underline{29}$, based on investigation of the discontinuities of some (properly constructed) SFs on the complex frequency plane. It is 
shown that the transition time increases considerably with the density decrease. It requires some tens of the (effective) particles collisions for the long-time tails formation that agrees completely with results of Refs. $\frac{12,27,41}{2}$. At this issue, our result is also consistent with the MCT viewpoint $\underline{30}, 42$, which considers the sequence of correlated collisions as a microscopic precursor of the long-time tails appearance.

An occurrence of the cut-off frequency $\omega_{c}$, which modulates the power law dynamics of the fluid at long times, can be explained in terms of the generalized Green-Kubo relations ${ }^{4.33}$. There is a narrowing of the dissipation channel in the high frequency domain $\frac{31}{}$, which cannot compensate a persistent renormalization of the vibrational modes by a non-vanishing imaginary part of the generalized self-diffusion coefficient.

It should be emphasized that a more accurate calculation of the high order memory kernels, presumably based on the infinite periodic continued fractions, is strongly desirable, because they are expected to ensure a smoother relaxation of the corresponding VAFs at long times, and to bring the long-time characteristics of the fluid much closer to those of the MCT prediction. Such a method requires not only a greater number of the relevant SCFs, which have to be taken from computer simulations, but also implies a very precise design of the higher order kinetic kernels for all the necessary sum rules to be satisfied. We believe that this task is quite promising and worthy the efforts to be spent to solve the problem how to describe the many-particle system dynamics on the whole time domain in the framework of a unified self-consistent approach.

\section{ACKNOWLEDGEMENT}

This study was partially supported within the project of the European Unions Horizon 2020 research and innovation programme under the Marie Skłodowska-Curie grant agreement No 734276.

\section{REFERENCES}

${ }^{1}$ E. Wild, Math. Proc. Camb. Philos. Soc. 47, 602 (1951).

${ }^{2}$ P.L. Bhatnagar, E.P. Gross, and M. Krook, Phys. Rev. 94, 511 (1954). 
${ }^{3}$ N.N. Bogolyubov, Problems of Dynamic Theory in Statistical Physics, (Technical Information Service, Oak Ridge, Tenn., 1960).

${ }^{4}$ D. Zubarev, V.G. Morozov, G. Roepke, Statistical Mechanics of Nonequilibrium Processes, Vol. 1, Basic Concepts, Kinetic Theory, (Akademy Verlag, Berlin, 1996).

${ }^{5}$ I.M. de Schepper, E.G.D. Cohen, C. Bruin, J.C. van Rijs, W. Montfrooij, and L.A. de Graaf, Phys. Rev. A 38, 271 (1988).

${ }^{6}$ I.P. Omelyan, I.M. Mryglod, Condens. Matter Phys. 4, 128 (1994).

${ }^{7}$ I.M. Mryglod, I.P. Omelyan, M.V. Tokarchuk, Mol. Phys. 84, 235 (1995).

${ }^{8}$ T.M. Bryk, I.M. Mryglod, A.D. Trokhymchuk, Condens. Matter Phys. 6, 23 (2003).

${ }^{9}$ I.M. Mryglod, R. Folk, Physica 234, 129 (1996).

${ }^{10}$ I.P. Omelyan, I.M. Mryglod, and M.V. Tokarchuk, Phys. Rev. E, 57, 6667 (1998).

${ }^{11}$ F. Barocchi, U. Bafile, and M. Sampoli, Phys. Rev. E. 85, 022102 (2012).

${ }^{12}$ S. Bellissima, M. Neumann, E. Guarini, U. Bafile, and F. Barocchi, Phys. Rev. E. 92, 042166 (2015).

${ }^{13}$ S. Bellissima, M. Neumann, E. Guarini, U. Bafile, and F. Barocchi, Phys. Rev. E. 95, 012108 (2017).

${ }^{14}$ I.P. Omelyan and M.V. Tokarchuk, J. Phys.: Condens. Matt. 12, L505 (2000).

${ }^{15}$ I.P. Omelyan, I.M. Mryglod, M.V. Tokarchuk, Condens. Matter Phys. 8, 25 (2005).

${ }^{16}$ T. Bryk and I. Mryglod, Phys. Rev. B 82, 174205 (2010).

${ }^{17}$ T. Bryk, I. Mryglod, Condens. Matter Phys. 11, 139 (2008).

${ }^{18}$ K. Kawasaki, Phys. Lett. A 32, 379 (1970).

${ }^{19}$ J. Bosse, W.Götze, and M. Lücke, Phys. Rev. A 17, 434 (1978).

${ }^{20}$ V.V. Ignatyuk, Condens. Matter Phys. 2, 37 (1999).

${ }^{21}$ V.G. Morozov, Physica A 117, 511 (1983).

${ }^{22}$ W. Götze, Phys. Scr. 34, 66 (1986).

${ }^{23}$ W. Götze, L. Sjögren, Chem. Phys. 212, 47 (1996).

${ }^{24}$ W. Götze, Complex Dynamics of Glass-Forming Liquids: A Mode-Coupling Theory (Oxford: University Press, 2009).

${ }^{25}$ G. Szamel, Phys. Rev. Lett. 90, 228301 (2003).

${ }^{26}$ L.M.C. Janssen, P. Mayer, and D.R. Reichman, Phys. Rev. E 90, 052306 (2014).

${ }^{27}$ D. Lesnicki, R. Vuilleumier, A. Carof, and B. Rotenberg, Phys. Rev. Lett. 116, 147804 (2016). 
${ }^{28}$ N.M. Chtchelkatchev, and R.E. Ryltsev, Singularity band of velocity auto correlation function of Lennard-Jones fluid in complex $\omega$-plain, arXiv:1507.04532v1 [cond-mat.soft].

${ }^{29}$ N.M. Chtchelkatchev, and R.E. Ryltsev, JETP Lett. 102, 643 (2015).

${ }^{30}$ B.J. Alder, and T.E. Wainwright, Phys. Rev. A 1, 18 (1970).

${ }^{31}$ V.V. Ignatyuk, I.M.Mryglod, T.Bryk, Condens. Matter Phys. 21, 13001 (2018).

${ }^{32}$ I.R. Yukhnovskii, M.F. Holovko, Statistical theory of classical equilibrium systems (Naukova Dumka, Kiev, 1980), [in Russian].

${ }^{33}$ J.-P. Boon and S. Yip, Molecular Hydrodynamics (New-York: McGraw-Hill) (1980).

${ }^{34}$ H. Mori, Prog. Theor. Phys. 33, 423 (1965); 34, 399 (1965).

${ }^{35}$ I.M. Mryglod, Condens. Matter Phys. 1, 753 (1998).

${ }^{36}$ L.M.B.C. Campos, Transcendental Representations with Applications to Solids and Fluids, (CRC Press, 2012).

${ }^{37}$ I.M. Mryglod, and A.M. Hachkevych, Ukr. Phys. J. 44, 901 (1999), [in Ukrainian].

${ }^{38}$ A. Roy, A. Dhar, O. Narayan, S. Sabhapandit, J. Stat. Phys. 160, 73 (2015).

${ }^{39}$ T. Bryk, F.A. Gorelli, I. Mryglod, G. Ruocco, M. Santoro, and T. Scopigno, J. Phys. Chem. Lett 8, 4995 (2017).

40 http://webbook.nist.gov/chemistry/fluid/

${ }^{41}$ R.E. Ryltsev, N.M. Chtchelkatchev, Journ. Chem. Phys. 141, 124509 (2014).

${ }^{42}$ D.R. Reichman, P. Charbonneau, J. Stat. Mech., P05013 (2005). 